\title{
Remarks on Oscillation of Second Order Differential Equations
}

\author{
A. G. Kartsatos* and Hiroshi OnOSE**
}

\section{Introduction.}

In this paper we shall discuss oscillatory properties of solutions of second order differential equations of the form

$$
y^{\prime \prime}+f\left(t, y, y^{\prime}\right)=0,
$$

and more general functional differential equations of the form

$$
y^{\prime \prime}+f\left(t, y\left(\delta_{1}(t)\right), y^{\prime}\left(\delta_{2}(t)\right)\right)=0,
$$

where $f(t, y, u)$ is continuous on $R_{+} \times R \times R, R_{+}=(0, \infty), R=(-\infty$, $\infty)$ and $\delta_{i}(t)(i=1,2)$ are continuous on $R_{+}$.

We consider only extendable solutions of (2). A solution of (2) is said to be oscillatory if it has arbitrarily large zeros, and (2) is said to be oscillatory if all of its solutions are oscillatory. For the special cases

$$
y^{\prime \prime}+a(t) f(y)=0,
$$

and

$$
y^{\prime \prime}+a(t) f(y(\delta(t)))=0,
$$

there exist numerous results. The reader is referred, for example, to Atkinson [1], Kartsatos [5], [6], Wong [11], Burkowski [2], Bradley $[3]$, and their references.

Received October 12, 1972.

*Department of Mathematics, University of South Florida, Tampa, Florida 33620, U. S. A. .

**Department of Mathematics, College of General Education, Ibaraki University, Mito. 
In the present note, we shall extend some results which have been obtained for $\left(1^{\prime}\right)$ and $\left(2^{\prime}\right)$ to the more general equations (1) and (2) respectively. The merit of this note lies also in the fact that our proofs are straightforward and simpler than the existing ones for the cases $\left(1^{\prime}\right),\left(2^{\prime}\right)$.

Theorem 1 generalizes some results of Coles [4]. Theorem 2 is a generalization of results of Kartsatos [5], Onose [7], [8].

Theorem 3 extends a recent result of Travis [10] and Onose, one of the authors, thanks Professor T. Kusano for his suggestions to Theorem 3.

Theorem 4 generalizes a result of Burkowski [2], and our proof is different.

We make use of the following conditions:

(i) for $t \geqq 0$ and $y \geqq 0$, there exist continuous functions $a(t)$ and $\alpha(y)$ such that $\lim _{t \rightarrow \infty} \inf \int_{T}^{t} a(s) d s \geqq 0$ for all large $T, y \alpha(y)>$ $0(y \neq 0), \quad \alpha^{\prime}(y) \geqq 0$, and for all large $t, y \geqq 0,|u|<\infty$, $a(t) \alpha(y) \leqq f(t, y, u)$;

(ii) for $t \geqq 0$ and $y \leqq 0$, there exist continuous functions $b(t)$ and $\beta(y)$ such that $\lim _{t \rightarrow \infty} \inf \int_{T}^{t} b(s) d s \geqq 0$ for all large $T$, and $y \beta(y)>0(y \neq 0), \beta^{\prime}(y) \geqq 0$, and for all large $t, y \leqq 0,|u|<\infty$, $f(t, y, u) \leqq b(t) \beta(y)$;

(iii) $\left.\quad \int_{0}^{\infty} a(s) d s<\infty, \int_{0}^{t} c \int_{s}^{\infty} a(u) d u\right) d s \rightarrow \infty \quad$ as $t \rightarrow \infty$,

$\int_{0}^{\infty} b(s) d s<\infty, \quad \int_{0}^{t}\left(\int_{s}^{\infty} b(u) d u\right) d s \rightarrow \infty \quad$ as $t \rightarrow \infty$,

$\int_{\varepsilon}^{\infty} \frac{d u}{\alpha(u)}<\infty \quad$ for some $\varepsilon>0$,

and

$\int_{-\varepsilon}^{-\infty} \frac{d u}{\beta(u)}<\infty \quad$ for some $\varepsilon>0$ 
(iv) $\int_{0}^{\infty} a(s) d s=\infty$, and $\int_{0}^{\infty} b(s) d s=\infty$;

(v) $\delta_{i}(t)(i=1,2)$ are continuous on $[a, \infty)$ and $\delta_{i}(t) \rightarrow \infty(i=1$, 2 ), as $t \rightarrow \infty$.

\section{Main theorems.}

THEOREM 1. (I) Let $f(t, y, u)$ satisfy the conditions (i), (ii), (iii). Then equation (1) is oscillatory.

(II) Let $f(t, y, u)$ satisfy the conditions (i), (ii), (iv). Then equation (1) is oscillatory.

Proof. Let $y$ be a nonoscillatory solution of (1) and assume that $y>0$ for some $q>0$ on $[q, \infty)$; a similar argument treats the case $y<0$.

Proof of (I):

From (1) and (i), we have $y^{\prime \prime}(t)=-f\left(t, y, y^{\prime}\right) \leqq-a(t) \alpha(y)$, $y^{\prime \prime}(t) / \alpha(y) \leqq-a(t)$. By integration from sufficiently large $c(\geqq q)$ to $t \geqq c$, we obtain

$$
\int_{c}^{t} \frac{y^{\prime \prime}(t)}{\alpha(y)} d t \leqq-\int_{c}^{t} a(t) d t
$$

By integration of (3) by parts, we have

$$
\left[y^{\prime}(t) / \alpha(y)\right]_{c}^{t}-\int_{c}^{t} \frac{y^{\prime}\left(-\alpha^{\prime}(y) y^{\prime}\right)}{\alpha^{2}(y)} d t \leqq-\int_{c}^{t} a(t) d t,
$$

so that

$$
\frac{y^{\prime}(t)}{\alpha(y(t))} \leqq \frac{y^{\prime}(c)}{\alpha(y(c))}-\int_{0}^{t} a(t) d t
$$

Let $y^{\prime}(t) \geqq 0$ for all large $t$, say $t \geqq d$. For $t \geqq d$ (4) gives

$$
\int_{t}^{\infty} a(s) d s \leqq \frac{y^{\prime}(t)}{\alpha(y(t))} .
$$

An integration from $d$ to $t \geqq d$, yields 


$$
\int_{d}^{t}\left(\int_{s}^{\infty} a(u) d u\right) d s \leqq \int_{d}^{t} \frac{y^{\prime}(s)}{\alpha(y(s))} d s=\int_{y(d)}^{y(t)} \frac{d y}{\alpha(y)}<\infty .
$$

This is a contradiction.

If $y^{\prime}(t)$ oscillates, then there exists $\left\{c_{n}\right\}$ such that $c_{n} \rightarrow+\infty$, as $c_{n} \rightarrow \infty$ and $y^{\prime}\left(c_{n}\right)<0, n=1,2, \ldots$ Take $c_{n}$ so large that $\int_{t}^{\infty} a(s) d s \geqq 0$ for $t \geqq c_{n}>q$. By (4),

$$
\frac{y^{\prime}(t)}{\alpha(y(t))} \leqq \frac{y^{\prime}\left(c_{n}\right)}{\alpha\left(y\left(c_{n}\right)\right)}-\int_{c_{n}}^{t} a(s) d s, \text { and } \int_{c_{n}}^{t} a(s) d s \geqq 0
$$

for sufficiently large $t$, say $t \geqq T \geqq c_{n}$. Hence, we conclude

$$
y^{\prime}(t)<0 \text { for all } t \geqq T \text {. }
$$

In this case we have

$$
\begin{aligned}
& \int_{T}^{t} a(s) \alpha(y(s)) d s=\alpha(y(t)) \int_{T}^{t} a(s) d s-\int_{T}^{t} \alpha^{\prime}(y(s)) y^{\prime}(s) \times \\
& \int_{T}^{s} a(u) d u d s \geqq 0
\end{aligned}
$$

for sufficiently large $t$, say $t \geqq k \geqq T$. By (1),

$$
y^{\prime \prime}(t)=-f\left(t, y, y^{\prime}\right) \leqq-a(t) \alpha(y(t)) .
$$

Intergrating (6) from $T$ to $t \geqq T$ we obtain $y^{\prime}(t)-y^{\prime}(T) \leqq$ $-\int_{T}^{t} a(s) \alpha(y(s)) d s \leqq 0$, for all $t \geqq k$, so that $y^{\prime}(t) \leqq y^{\prime}(T)<0$ for all $t \geqq k$, which implies $y(t)<0$ for all sufficiently large $t$. This is a contradiction.

Proof of (II):

By (iv) and (2) we have at once $y^{\prime}(t) / \alpha(y(t)) \rightarrow-\infty$ as $t \rightarrow \infty$. Hence we obtain $y^{\prime}(t)<0$ for sufficiently large $t$. Thus, we have at once a contradiction as in the proof of Case 2 of (I).

Q. E. D.

THEOREM 2. Let $f$ satisfy the conditions (i), (ii), and

$$
\int_{x}^{\infty} s a(s) d s=+\infty, \quad \int_{x}^{\infty} s b(s) d s=+\infty,
$$




$$
\int_{\varepsilon}^{\infty} \frac{d u}{\alpha(u)}<\infty \text { and } \int_{-\mathrm{e}}^{-\infty} \frac{d u}{\beta(u)}<\infty \text {, for some } \varepsilon>0 \text {. }
$$

Then equation (1) is oscillatory.

PROOF. Let $y$ be nonoscillatory and assume that $y(t)>0$ for $t \in[p$, $+\infty$ ) for some $p>0$; a similar argument treats the case $y<0$. Then from (1) we obtain

$$
t y^{\prime \prime} / \alpha(y) \leqq-t a(t) .
$$

By integration from $p$ to $t \geqq p$ we obtain

$$
\frac{t y^{\prime}(t)}{\alpha(y(t))} \leqq \int_{v(p)}^{\nu(t)} \frac{d s}{\alpha(s)}-\int_{p}^{t} s a(s) d s+\frac{p y^{\prime}(p)}{\alpha(y(p))},
$$

which implies that

$$
\frac{t y^{\prime}(t)}{\alpha(y(t))} \rightarrow-\infty, \text { as } t \rightarrow \infty
$$

So that we have

$$
y^{\prime}(t)<0 \text { for all sufficiently large } t .
$$

By the assumption (i), we may suppose that $T_{1}$ is sufficiently large so that

$$
\int_{T_{1}}^{t} a(s) d s \geqq 0 \text { and } y^{\prime}(t)<0,
$$

for all $t \geqq k \geqq T_{1}$. Hence, we have

$$
\begin{aligned}
& \int_{T_{1}}^{t} a(s) \alpha(y(s)) d s=\alpha(y(t)) \int_{T_{1}}^{t} a(s) d s- \\
& \quad \int_{T_{1}}^{t} \alpha^{\prime}(y(s)) y^{\prime}(s) \int_{r_{1}}^{s} a(u) d u d s \geqq 0, t \geqq k .
\end{aligned}
$$

By (1) we have

$$
y^{\prime}(t)-y^{\prime}\left(T_{1}\right)+\int_{T_{1}}^{\iota} a(s) \alpha(y(s)) d s \leqq 0,
$$


so that $y^{\prime}(t) \leqq y^{\prime}\left(T_{1}\right)<0, t \geqq k$, which implies $y(t)<0$ for all large t. This contradicts $y(t)>0$.

Q. E. D.

In what follows, we consider a functional differential equation of type (2).

THEOREM 3. Let $f(t, y, u)$ satisfy the conditions (i), (ii), (iii), $(v)$, and $\delta_{1}^{\prime}(t) \geqq 0$. Then, $y^{\prime}(t)$ is oscillatory for any solution of (2).

Proof. Suppose $y^{\prime}(t)$ is not oscillatory. If $y(t)$ oscillates, then $y^{\prime}(t)$ oscillates, which leads to a contradiction. Hence, we suppose $y(t)$ is ultimately positive (the case $y(t)<0$ can be treated similarly). Then so is $y\left(\delta_{1}(t)\right)$ and $\alpha\left(y\left(\delta_{1}(t)\right)\right)$. Let

$W(t)=y^{\prime}(t) / \alpha\left(y\left(\delta_{1}(t)\right)\right)$. Then we have

$$
W^{\prime}(t) \leqq-a(t)-\left\{y^{\prime}(t) y^{\prime}\left(\delta_{1}(t)\right)\right\} \alpha^{\prime}\left(y\left(\delta_{1}(t)\right)\right) \delta_{1}^{\prime}(t) / \alpha\left(y\left(\delta_{1}(t)\right)\right)^{2} .
$$

Since $y^{\prime}(t)$ is not oscillatory, we have $y^{\prime}(t) y^{\prime}\left(\delta_{1}(t)\right)>0$ for sufficie ntly large $t$. Thus, $W^{\prime}(t) \leqq-a(t)$. Integrating from $c$ to $x \geqq c$, we obtain

$$
W(x) \leqq W(c)-\int_{c}^{x} a(t) d t
$$

By (iv), we have

(11) $y^{\prime}(t)<0$, for all sufficiently large $t$. Consequently,

$$
\begin{aligned}
& \int_{T}^{t} a(s) \alpha\left(y\left(\delta_{1}(s)\right)\right) d s=\alpha\left(y\left(\delta_{1}(t)\right)\right) \int_{T}^{t} a(s) d s \\
& \quad-\int_{T^{\prime}}^{t} \alpha^{\prime}\left(y\left(\delta_{1}(t)\right)\right) y^{\prime}\left(\delta_{1}(t)\right) \delta_{1}^{\prime}(t) \int_{T}^{s} a(u) d u d s \geqq 0 .
\end{aligned}
$$

By (2), $y^{\prime \prime} \leqq-a(t) \alpha\left(y\left(\delta_{1}(t)\right)\right.$. By integration from $T$ to $t \geqq T$, we have at once $y^{\prime}(t) \leqq y^{\prime}(T)<0$. Hence $y(t)<0$ for sufficiently large $t$. This contradicts the fact $y(t)>0$. We, conclude that $y^{\prime}(t)$ is oscilla tory.

Q. E. D.

THEOREM 4. (I) Let $f(t, y, u)$ satisfy the conditions (i), (ii), (iv), (v), and let $\delta_{1}(t)$ be differentiable with $0 \leqq \delta_{1}^{\prime}(t) \leqq 1$. Finally 
assume that $a(t) \geqq 0$. Then equation (2) is oscillatery.

(II) In (I), suppose (iii) instead of (iv). In addition to the hypotheses of $(\mathrm{I})$, assume that $\delta_{i}(t)=t-\tau_{i}(t), 0<\tau_{i}(t) \leqq M(i=1,2)$, where $\tau_{i}(t)$ is continuous. Then equation (2) is oscillatory.

PROOF. (I) :

Suppose $y(t)$ is ultimately positive (the case $y(t)<0$ can be treated similarly). Then, by (2),

$$
y^{\prime \prime}=-f\left(t, y\left(\delta_{1}(t)\right), y^{\prime}\left(\delta_{2}(t)\right)\right) \leqq-a(t) \alpha\left(y\left(\delta_{1}(t)\right)\right) \leqq 0 .
$$

Hence we have $y^{\prime}(t)>0$ for all sufficiently large $t$. Let $W(t)=y^{\prime}(t)$ $/ \alpha\left(y\left(\delta_{1}(t)\right)\right)$. Then $W^{\prime}(t) \leqq-a(t)$. Integrating it, we obtain

$$
W(x) \leqq W(c)-\int_{c}^{x} a(t) d t .
$$

Because of the assumption (iv), we have $y^{\prime}(t)<0$ for all sufficiently large $t$. This is a contradiction. Hence (I) is proved.

(II) :

By the same argument as in Staikos and Petsoulas [9],

$$
\begin{aligned}
\left|\frac{y\left(t-\tau_{1}(t)\right)}{y(t)}-1\right| & =\frac{y(t)-y_{1}(t-\tau(t))}{y(t)} \\
& \leqq \frac{y(t)-y(t-M)}{y(t)}
\end{aligned}
$$

and by the mean value theorem,

$$
\frac{y(t)-y(t-M)}{y(t)}=\frac{M y^{\prime}(\xi)}{y(t)} \leqq M \frac{y^{\prime}(t-M)}{y(t)} \leqq M \frac{y^{\prime}(t-M)}{y(t-M)} .
$$

Thus, we obtain

$$
\text { (13) }\left|\frac{y\left(t-\tau_{1}(t)\right)}{y(t)}-1\right|<M \frac{y^{\prime}(t-M)}{y(t-M)}, \quad t \in\left[t_{0}+M,+\infty\right) \text {. }
$$

After a simple computation, we have 


$$
\lim _{t \rightarrow \infty} \frac{y^{\prime}(t)}{y(t)}=0
$$

hence, by (13), we obtain

$$
\lim _{t \rightarrow \infty} \frac{y\left(\delta_{1}(t)\right)}{y(t)}=1
$$

By (14), for $t \geqq T$ (sufficiently large), we have

$$
\frac{y\left(\delta_{1}(t)\right)}{y(t)}>\frac{1}{2} \text { or } y\left(\delta_{1}(t)\right)>\frac{1}{2} y(t)
$$

If (iii), then (12) and (15) give

$$
\int_{t}^{\infty} a(s) d s \leqq \frac{y^{\prime}(t)}{\alpha\left(y\left(\delta_{1}(t)\right)\right)} \leqq \frac{y^{\prime}(t)}{\left(\frac{1}{2} y(t)\right)} .
$$

Integrating it from $d$ to $t \geq d$, we have

$$
\begin{aligned}
\int_{a}^{t}\left(\int_{s}^{\infty} a(u) d u\right) d s & \leqq \int_{a}^{t} \frac{y(s)}{\left(\frac{1}{2}-y(s)\right)} d s \\
& =2 \int_{\frac{1}{2} y(d)}^{\frac{1}{2} v(t)} \frac{d s}{\alpha(s)}<\infty .
\end{aligned}
$$

Which leads to a contradiction. Hence(II) is proved.

Q. E. D.

\section{References}

[1] F. V. Atkinson, On second order non-linear oscillation, Pacific J. Math., 5 (1955), 643-647.

[2] F. Burkowski, Oscillation theorems for a second order non-linear functional differential equations, J. Math. Anal. Appl,, 33 (1971), 258-262.

[3] J. S. Bradley, Oscillation theorems for a second-order delay equation, J. Differential Equations, 8 (1970), 397-403.

[4] W. J. Coles, Oscillation criteria for non-linear second order equations, Ann. Mat. Pure appl., 82 (1969), 123-133. 
[5] A. G.Kartsatos, Properties of bounded solutions of nonlinear equations of second order, Proc. Amer. Math. Soc., 19 (1968), 1057-1059.

[6] G. G. Legatos and A. G. Kartsatos, Further results on oscillations of second order equations, Math. Japo., 14 (1968), 67-73.

[7] H. Onose, Oscillation theorems for nonlinear second order differential equations, Proc. Amer. Math. Soc., 26 (1970), 461-464.

[8] H. Onose, On oscillation of nonlinear second order equations, J. Math. Anal Appl. (1972).

[9] V. A. Staikos and A. G. Petsoulas, Some oscillation criteria for second order nonlinear delay-differential equations, J. Math. Anal. Appl., 30 (1970), 695-701.

[10] C. C. Travis, Oscillation theorems for second-order differential equations with functional argments, Proc. Amer. Math. Soc., 31 (1972), 199-202.

[11] J. S. W. Wong, On second order nonlinear oscillation, Funk. Ekvac., 11(1968), 207-234. 